\title{
The inflation response of the posterior bovine sclera
}

Kristin M. Myers ${ }^{\mathrm{a}}$, Baptiste Coudrilliera, Brad L. Boyceb, Thao D. Nguyen ${ }^{\mathrm{a}}$

a Department of Mechanical Engineering, Johns Hopkins University, Baltimore, MD 21218, USA

b Materials Science and Engineering Center, Sandia National Laboratories, Albuquerque, NM 87185, USA

\section{Introduction}

The eye wall is the primary structural component of the eye, and it consists of a transparent cornea and opaque sclera. Both tissues serve to protect the delicate internal ocular structures from external injuries and to preserve the shape of the refractive components from changes in the intraocular pressure (IOP). The sclera is a hydrated structure containing densely stacked lamellae of type I collagen fibrils. The collagen structure has a spatially varying predominant orientation, which may be dictated by the optic nerve head $(\mathrm{ONH})$ in the peripapillary region [1]. The collagen lamellae are embedded in a highly viscous proteoglycan ground substance with scleral fibroblasts sparsely dispersed in the tissue [2]. Elastin has also been found in human and animal scleral tissue in the peripapillary region surrounding the $\mathrm{ONH}[3]$ and [4]. This extracellular matrix (ECM) structure provides the sclera with the mechanical stiffness and strength needed to maintain an optimal ocular shape for vision and to mechanically support the $\mathrm{ONH}$. Conditions and diseases such as myopia and glaucoma have been associated with aberrant scleral ECM structure and mechanical properties [4] and [5]. Glaucoma is a neurodegenerative condition caused by the progressive loss of the retinal ganglion cells (RGCs) [6] and is characterized by excessive cupping and excavation of the ONH [7]. The level of IOP is a strong risk factor for the disease, and it is hypothesized that excessive mechanical stress in the sclera generated by the level of IOP precipitates a cascade of biochemical and mechanical processes that leads to RGC apoptosis and glaucomatous damage [7], [8] and [9]. Myopia, or nearsightedness, is produced by a lengthening of the eye, which moves the retina behind the focus plane. High myopia is associated with the thinning of the sclera and changes in the viscoelastic properties and ECM structure of the tissues [10], [11] and [12]. Mechanical characterization of the sclera is needed to understand the development of these ocular diseases and to aid in early diagnosis and treatment.

In this paper, we present the development of a protocol for the inflation testing of the posterior sclera, which measures the spatially resolved three-dimensional (3-D) instantaneous displacement field of the tissue in response to increases in pressure. This work presents the application of this method to the characterization of the structural inflation behavior of the bovine sclera. Previous in vitro mechanical experiments on scleral tissue, from humans and animal models, include uniaxial tensile tests on strip specimens excised from intact sclera [11], [13], [14], [15], [16], [17] and [18] and inflation tests on intact tissue specimens [12], [19], [20] and [21]. Previous in vivo mechanical studies 
include human ocular rigidity tests [22] and ocular creep tests on animal models [23]. Through uniaxial testing it was found that the mechanical properties of scleral tissue were nonlinear, heterogeneous, anisotropic and viscoelastic. Further, it was found that material properties were significantly different between normal specimens and specimens with induced glaucoma [14] and induced myopia [10] and [11]. Uniaxial strip tests provide an accessible method to measure and compare material behavior. However, the uniaxial loading and associated boundary conditions do not represent the complex in vivo mechanical loading environment. In addition, the specimen preparation process likely alters the collagen fibril structure and disrupts the natural curvature of the tissue. The former may cause the tissue to soften during the repeated loading cycle of preconditioning [13], [14], [15] and [16].

Hommer et al. [22] measured the in vivo ocular expansion of human eyes induced by blood pressure pulsation and reported that the eyes of glaucoma patients exhibited a higher estimated ocular stiffness than those of non-glaucoma patients. Phillips and McBrien [23] measured the change in axial length via ultrasound in cannulated and anesthetized chick and tree shrew eyes. The results showed the eyes of both animals exhibited an instantaneous elastic response to an increase in IOP from 15 to $100 \mathrm{mmHg}$. At $100 \mathrm{mmHg}$, the axial length elongated with time for chick eyes but decreased slightly with time for those of tree shrews. The difference in the in vivo creep behavior between the two species was attributed to the presence of active contractile myofibroblasts in the tree shrew eyes, which function to maintain the ocular shape in vivo. In general, in vivo tests incorporate the effects of mechanisms that would be difficult to replicate in an in vitro experiment. However, in vivo experiments measure the mechanical response of the entire globe, which includes the contributions of the sclera, cornea and internal ocular components. In vitro methods allow the mechanical behavior of the sclera to be measured separately from those of the cornea and other ocular components.

Compared to in vitro strip tests, inflation tests require less sample preparation, which preserves the native collagen/elastin structure and provides for a more physiological representation of the in vivo loading conditions. Previous in vitro inflation tests on human [21], porcine [20], monkey [19] and rabbit scleral tissue [12] showed that the posterior sclera deformed nonlinearly in response to pressure elevation, stiffened with age [19] and crept over long periods of time in response to a constant elevated pressure. Our method improved on the most recent inflation tests of the sclera by measuring the time-resolved and spatially resolved 3-D scleral deformation in response to controlled pressurization, which enables the characterization of the viscoelastic and regional material properties of the tissue. This paper presents a robust and repeatable in vitro inflation method that captures the time-dependent and spatially varying mechanical response of the sclera to increases in pressure and its application to bovine sclera. Future work will apply the method to compare the inflation response of normal and diseased human sclera to study the role of biomechanics in the development of glaucoma and myopia. 


\section{Methods}

\subsection{Material}

Untreated left-right pairs of bovine eyes were harvested from animals younger than 30 months (Animal Technology, Inc., Tyler, TX). A set of specimens were used for preliminary investigations and protocol development, and a total of 10 eyes were tested using the viscoelastic testing regimen presented in Section 2.3. Specimens were shipped packed on ice, stored at $4{ }^{\circ} \mathrm{C}$ and tested within $72 \mathrm{~h}$ of slaughter. The specimens were not provided with culture medium to maintain the viability of the cells during storage. Therefore, some tissue degradation may have occurred. However, a previous study by Girard [14] using uniaxial tensile tests showed little difference in the viscoelastic material properties measured for fresh scleral tissue and tissue stored in PBS at $4{ }^{\circ} \mathrm{C}$ and tested within $72 \mathrm{~h}$ of enucleation. In addition, preliminary tests performed in our laboratory also showed a negligible difference in the inflation response of specimens tested within $72 \mathrm{~h}$ of slaughter. Each specimen was cleaned of orbital muscle and fat, with the ONH cut flush to the scleral surface, and kept moist by repeatedly dripping phosphate-buffered saline (PBS) during preparation.

\subsection{Specimen preparation}

The cleaned globes were glued to a custom-machined plastic ring from the saddle-shaped limbus to $10 \mathrm{~mm}$ posterior to the limbus using cyanoacrylate. The ring fixture was designed specifically to match the saddle-shape of the bovine limbus (Fig. 1A), and was described in a previous publication [24]. The locations of the nasal and temporal poles were marked on the fixture. The cornea was excised from the globe and the internal ocular components, including the choroid and the retina, were removed. To provide a rigid hold of the scleral specimen, the internal surface of the scleral rim was scored and impregnated with cyanoacrylate.
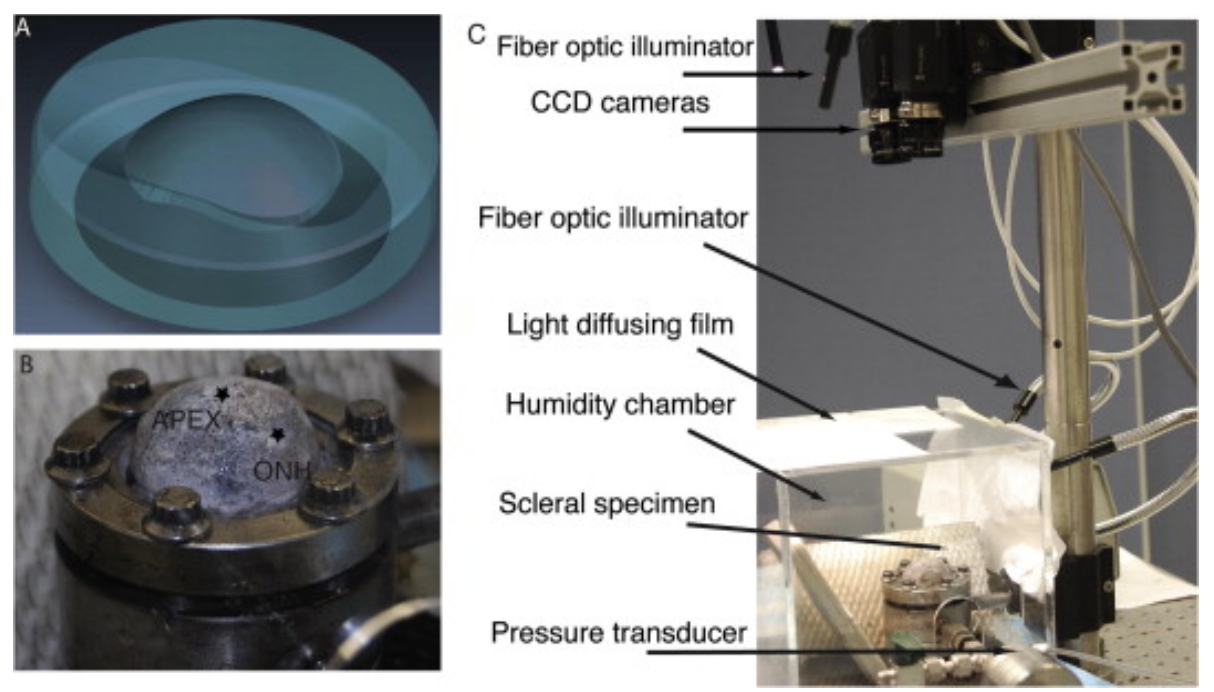

Fig. 1. (A) Saddle-shaped bovine eye inflation holder. Specimens were glued from the saddle-shaped limbus to $10 \mathrm{~mm}$ posterior to the limbus. For scleral inflation, the cornea was excised from the globe and the internal ocular components were removed. (B) Speckled scleral specimen in pressure chamber. (C) Experimental set-up. 
The specimen and holder were placed in a custom stainless steel pressure chamber (Fig. 1B). The design of the pressure chamber was described in a previous publication on inflation testing of bovine cornea [24]. Briefly, the pressure chamber consisted of three ports for (1) a pressure transducer (Honeywell, Sensing and Control, precision: $0.02 \mathrm{kPa}$ (0.15 $\mathrm{mm} \mathrm{Hg})$ ), (2) an inlet for fluid injection and (3) a release pressure valve. The inflation pressure was prescribed through active feedback control of an automated syringe injection system (MTS Insight, TestWorks 4). Specimens were discarded if leaking was detected during inflation.

To begin testing, the chamber was filled with isotonic PBS and pressurized to a baseline pressure of $0.21 \mathrm{kPa}(1.55 \mathrm{~mm} \mathrm{Hg})$. The baseline pressure was chosen as the lowest pressure under which the specimen could support its own weight without buckling. The baseline pressure represents the low-pressure end of the testing range. The tissue was allowed to equilibrate at the baseline pressure for $30 \mathrm{~min}$ before testing. During this time, the sclera was speckled with a black and white contrasting pattern for deformation tracking with the digital image correlation (DIC) system (see Section 2.4). To provide a sufficiently sharp contrast, the scleral surface was gently blotted dry, stained with white India ink and air brushed with black India ink. After the ink dried, the tissue was rehydrated by dripping PBS onto the surface. The specimen and inflation apparatus were enclosed in a humid environmental chamber (Fig. 1C) to maintain tissue hydration throughout testing. The humidity and temperature recorded for all the tests were $67 \pm 4 \%$ and $24 \pm 1{ }^{\circ} \mathrm{C}$. Preliminary tests performed without the humidity chamber showed the specimen stiffening during the hours-long test because of dehydration. The addition of the environmental chamber eliminated this stiffening behavior. Preliminary tests were also conducted to determine the effect of the India ink surface treatment on the inflation response. Specimens speckled with graphite powder and specimens stained and speckled with India ink were subjected to the same load-unload tests. The displacement response measured for both sets of specimens yielded indistinguishable behavior. The India ink speckling method provided better control of the particle size, which improved the resolution and reduced the correlation error of the DIC displacement measurements.

\subsection{Loading regimen}

A loading regimen was developed and applied to 10 specimens to characterize the viscoelastic behavior of the posterior sclera (Fig. 2). Each specimen was first subjected to three pressure linear load-unload cycles from the baseline pressure to $4.0 \mathrm{kPa}$ (30 $\mathrm{mmHg}$ ) at a rate of $0.13 \mathrm{kPa} \mathrm{s}^{-1} 1$ to determine the effects of preconditioning. The cycles were separated by a recovery period at the baseline pressure for $15 \mathrm{~min}$ to allow for full recovery after unloading. This was followed by two additional load-unload tests at a rate of $0.7 \mathrm{kPa}-1$ with a 20 -min recovery period and at $0.007 \mathrm{kPa} \mathrm{s}-1$ with a 30 -min recovery period at the baseline pressure. Full recovery was achieved after each loading cycle. The pressure range (1.55-30 mmHg) of the test was able to capture the full range of the nonlinear pressure-displacement response, including the compliant toe-region at low pressures, the stiffening heel region and the linear region at high pressures. Next, each 
specimen was subjected to three pressure ramp-hold tests to $2.0 \mathrm{kPa}(15 \mathrm{mmHg}), 4.0 \mathrm{kPa}$ ( $30 \mathrm{mmHg}$ ) and $6.0 \mathrm{kPa}$ ( $45 \mathrm{mmHg}$ ). Each ramp-hold test was loaded at a rate of $0.13 \mathrm{kPa} s$ -1 and separated by a 30-min recovery time at the baseline pressure. The tested pressures were within the physiological range for healthy and glaucomatous human eyes and typical bovine eyes [25]. The tested pressure rates spanned a broad range of values (two orders of magnitude), which likely included a large span of in vivo conditions. In addition, this large range of the tested pressure rates will allow for the improved characterization of the scleral viscoelastic behavior. To ensure the tissue did not degrade during testing, a final load-unload test from the baseline pressure to $30 \mathrm{mmHg}$ at a rate of $0.13 \mathrm{kPa} \mathrm{s}-1$ was performed for comparison with the first load-unload test.
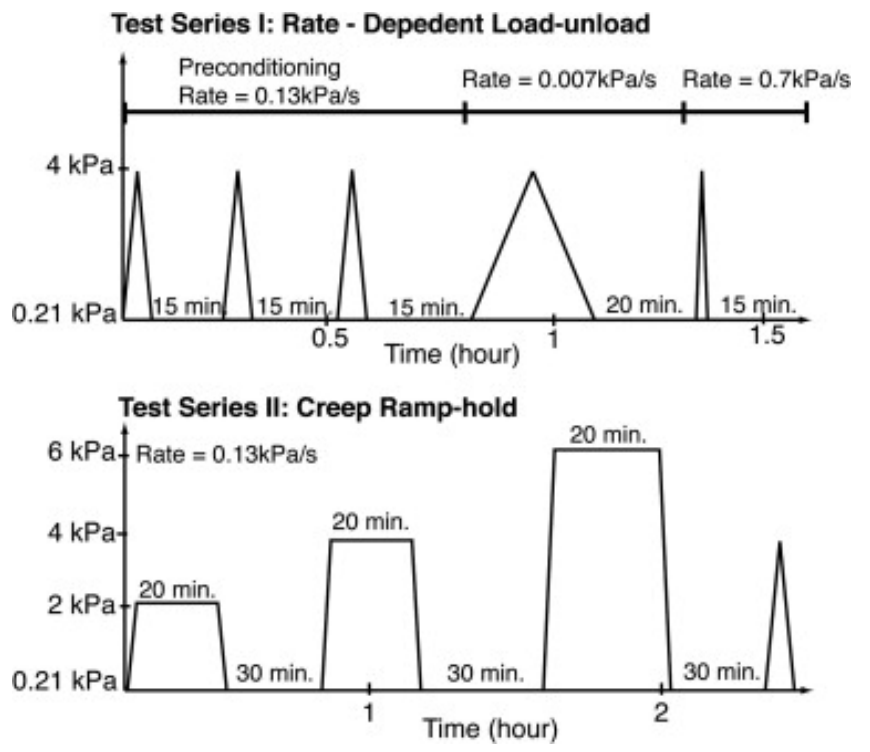

Fig. 2. Pressure-controlled testing segments.

After testing, the diameters of the nasal-temporal (NT) and inferior-superior (SI) meridians were measured with calipers in repeats of three at the equator as well as the posterior height from the specimen holder to the scleral apex. The specimen was then removed from the holder and cut along the nasal-temporal axis. The scleral thickness at the apex and the nasal equator were measured with calipers in repeats of three (Fig. 3). The averaged thickness, diameters and posterior height measurements are reported in Table 1 for 27 specimens. There was no significant difference between the measurements comparing the SI and NT diameters. However, the scleral tissue was significantly thicker at the posterior apex when compared to the equatorial region (Student' $s t$-test, $p<0.05$ ). It should be noted that diameter measurements were taken before and after testing, and the differences were negligible. 

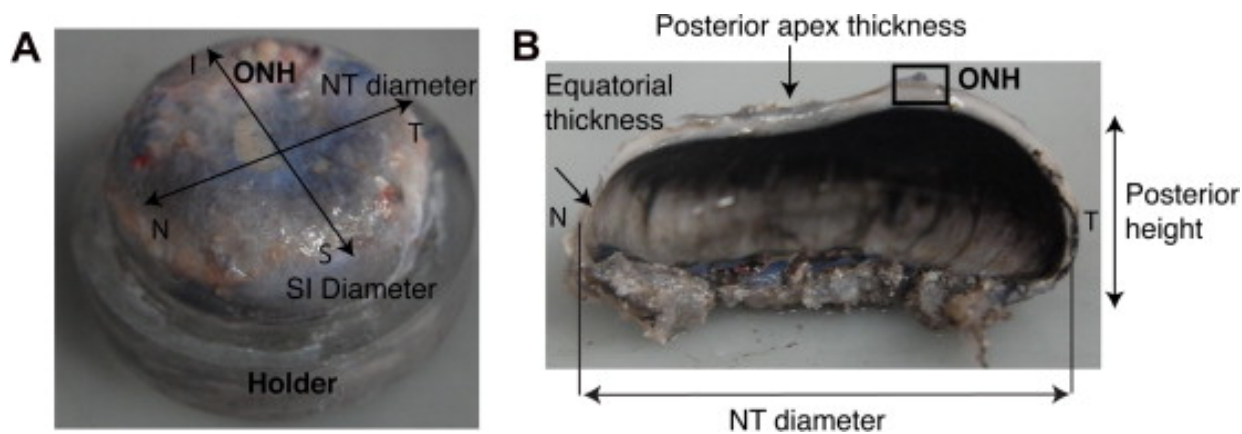

Fig. 3. (A) A scleral specimen glued to the holder showing the location of the $\mathrm{ONH}$ relative to the apex. (B) A cross-section of the scleral specimen (removed from the holder after testing) illustrating the locations of the thickness measurements and measurements of the posterior height and diameters.

Table 1. Principal dimensions of the sclera.

\begin{tabular}{l|l|l} 
& $\begin{array}{l}\text { Mean } \\
(\mathrm{mm})\end{array}$ & $\begin{array}{l}\text { Standard deviation } \\
(\mathrm{mm})\end{array}$ \\
\hline SI diameter & 36.85 & 1.50 \\
\hline NT diameter & 37.19 & 1.63 \\
\hline $\begin{array}{l}\text { Posterior height } \\
\begin{array}{l}\text { Posterior apex } \\
\text { thickness }\end{array}\end{array}$ & 19.52 & 1.19 \\
\hline Peripheral thickness & 1.10 & 0.39 \\
\hline
\end{tabular}

\subsection{Data acquisition and digital image correlation}

A stereoscopic DIC system was used to measure the spatially and time-resolved map of the displacement response of the scleral surface during inflation testing (see Boyce et al. [24] and Schreier [26] for a further discussion of stereoscopic DIC analysis). Two cameras (Pt. Grey Research GRAS-20S4M/C, Schneider Kreuznach $35 \mathrm{~mm}$ lens, f8) were positioned above the specimen and canted symmetrically at an angle of $15^{\circ}$ from the vertical axis. The specimen was illuminated with two fiber optic lamps coupled with light-diffusing film (Fig. 1C). Pictures of the deforming sclera were taken at a rate of $0.5 \mathrm{~Hz}$ (Vic-Snap, Correlated Solutions, Inc.) with a pixel resolution of $3.4 \mu \mathrm{m}$. Test image-pairs were analyzed using a 3-D DIC software package (VIC 3-D, Correlated Solutions, Inc.). The material points X, Y and $\mathrm{Z}$ were determined for the configuration of the scleral surface at the baseline pressure, with the $\mathrm{z}$-axis coinciding with the vertical axis. The displacements $\mathrm{U}$ (x-direction), $\mathrm{V}$ (ydirection) and $\mathrm{W}$ (z-direction) were then calculated by comparing the deformed and baseline images. The location and the optical range of the cameras captured the displacement field of the posterior sclera up to the equator.

The error of the DIC algorithm was estimated for each specimen. Before testing, two sets of stereo images of the scleral specimen were taken $1 \mathrm{~s}$ apart at the baseline pressure. A displacement map was generated by correlating the second image set with the first, and an error Ei was defined for each material point i using Eq. (1), where U, V and W are the X, Y 
and $\mathrm{Z}$ displacement components, respectively, and the indices 1 and 2 refer to the image set,

$E_{i}=\sqrt{\left(U_{i}^{2}-U_{i}^{1}\right)^{2}+\left(V_{i}^{2}-V_{i}^{1}\right)^{2}+\left(W_{i}^{2}-W_{i}^{1}\right)^{2}}$

Theoretically, Ei should be zero because the two images taken at the same pressure and under nominally identical conditions. A finite value of $\mathrm{Ei}$, reflects the inherent error of the DIC measurements. For a typical scleral specimen, the mean value of Ei was $6.8 \pm 2.7 \mu \mathrm{m}$, with the error in the central zone measuring on average $3.6 \mu \mathrm{m}( \pm 1.1 \mu \mathrm{m})$ and in the equatorial zone measuring on average $7.2 \mu \mathrm{m}( \pm 2.9 \mu \mathrm{m})$. We expected the error in the equatorial zone to be larger because of the inferior lighting conditions compared to the central zone.

\section{Results}

Fig. 1B shows a typical scleral specimen with the airbrush speckle pattern, while Fig. 4 shows the 3-D DIC reconstruction of the deformed specimen shape and displacement field of the specimen surface inflated to $4 \mathrm{kPa}(30 \mathrm{mmHg})$. The vertical component (W) dominated the displacement with values on the order of $1 \mathrm{~mm}$. For nearly all the specimens, the maximum vertical displacements were observed in the peripapillary region around the $\mathrm{ONH}$, which was located a distance away from the apex (Fig. 4B). Fig. 5 shows the evolution of the displacement magnitude from $0.21 \mathrm{kPa}(1.56 \mathrm{mmHg})$ to $4 \mathrm{kPa}$ (30 $\mathrm{mmHg}$ ) for a typical bovine scleral sample. By definition, the displacement magnitude is zero at the baseline pressure of $0.21 \mathrm{kPa}$. For a homogeneous, isotropic material, we would expect the contours to form concentric circles around the apex with the maximum displacement located at the apex. In our results, the maximum displacements were offcentered and located around the $\mathrm{ONH}$ in the peripapillary sclera. This displacement contour pattern could be the influence of material heterogeneity. Further modeling and histology studies are needed to investigate the deformation mechanisms of the sclera.

A
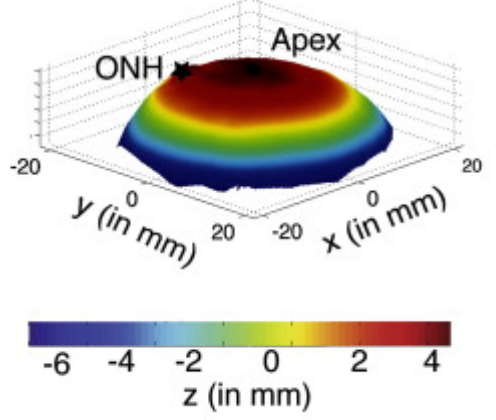

B
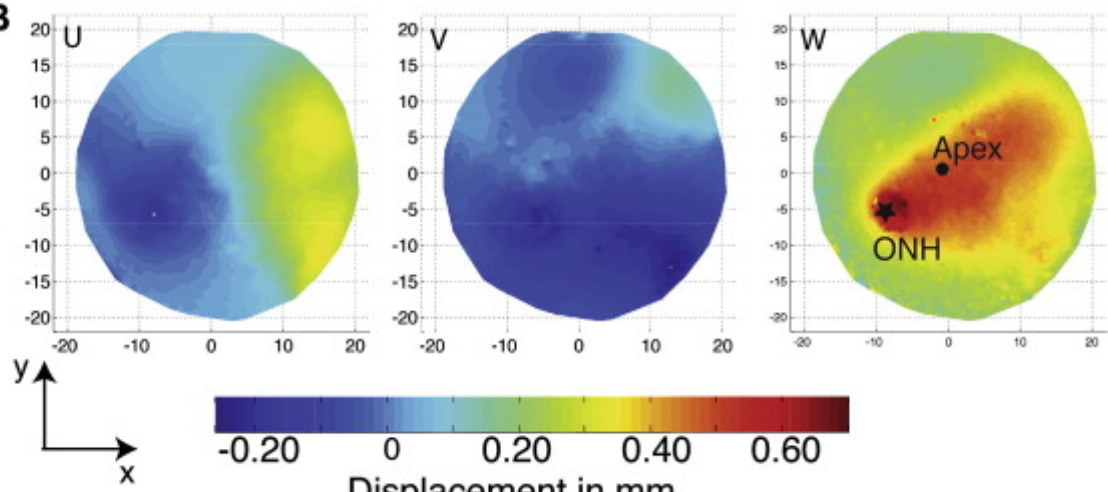

Fig. 4. (A) 3-D DIC reconstruction of the bovine scleral surface. (B) Contours of the displacement components $\mathrm{U}, \mathrm{V}$ and $\mathrm{W}$ for the $\mathrm{x}-, \mathrm{y}$ - and $\mathrm{z}$-directions, respectively. The vertical component $(W)$ dominated the displacement with values on the order of $1 \mathrm{~mm}$. 


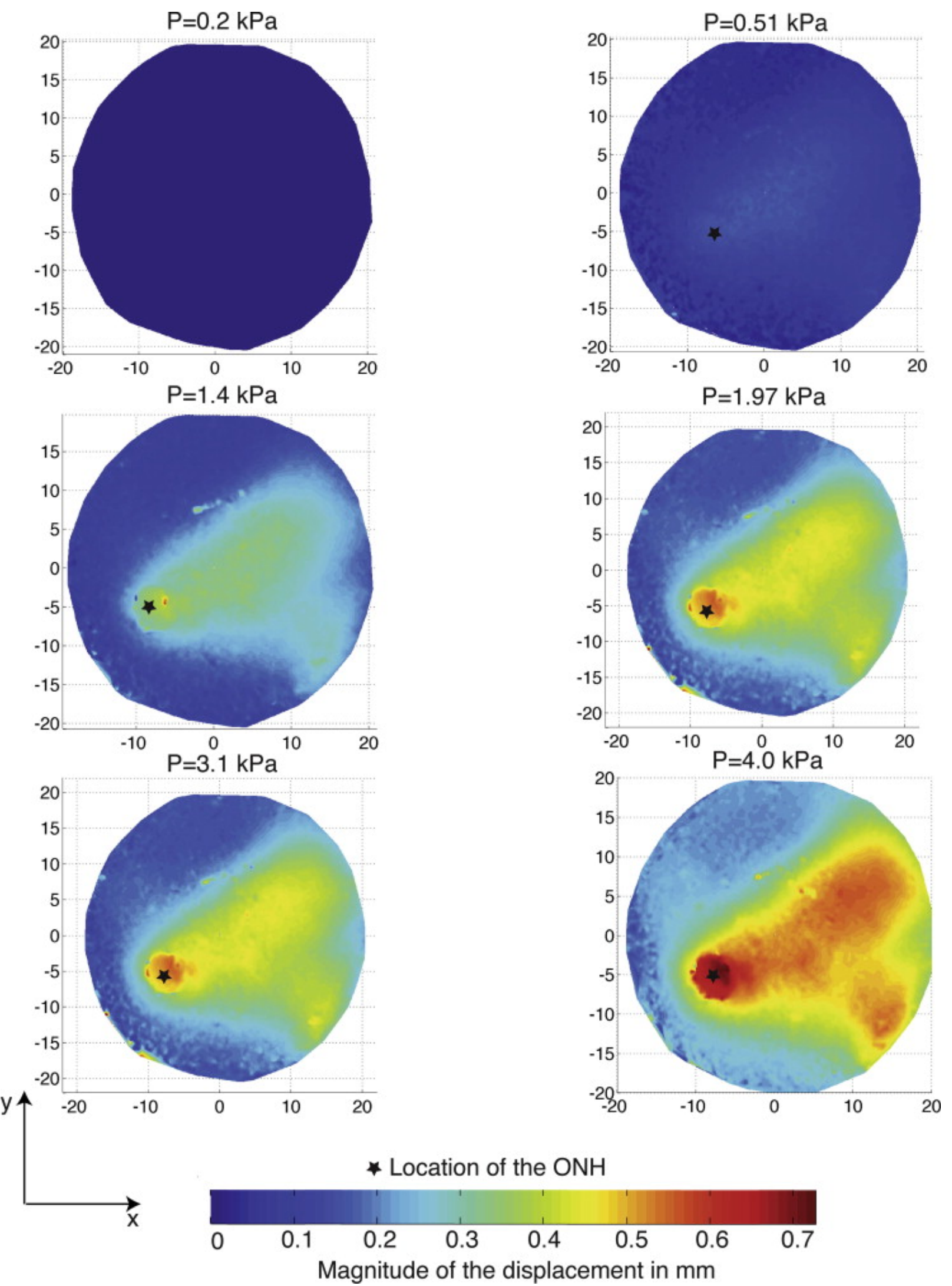

Fig. 5. The 3-D contour evolution of the magnitude of the displacement for a typical bovine 
scleral sample. The maximum displacements were off-centered and located around the $\mathrm{ONH}$ in the peripapillary sclera.

\subsection{Effects of preconditioning}

Fig. 6 compares the apex displacement response of the first three identical pressure cycles of the loading regimen (Fig. 2). The results are plotted for only three specimens spanning the measured variation in the displacement response for clarity, but these results were typical for all 10 specimens. The displacement increased nonlinearly with increasing pressure, displaying a J-shape typical of collagenous tissues. For each specimen, the standard deviation was calculated for the peak apex displacement measurements of the first three pressure cycles. The average standard deviation for the 10 specimens was $5 \mu \mathrm{m}$ and the maximum was $10 \mu \mathrm{m}$. These standard deviation measurements were on the order of the experimental error (calculated in Section 2.4). Thus, there was no significant difference measured for the displacement response between the first three cycles, which demonstrated that preconditioning had a negligible effect on the mechanical behavior of the tissues.

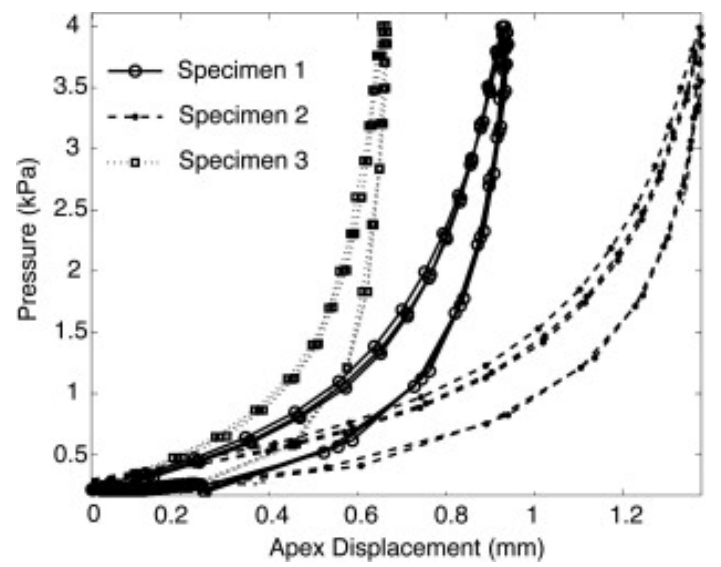

Fig. 6. Apex displacement response comparing the first three pressure cycles of the loading regimen for three specimens. There was no preconditioning effect measured for the tested specimens.

\subsection{Effects of recovery}

The loading regimen was designed to allow the deformation to completely recover between each pressure cycle and creep test. Fig. 7 compares the apex displacement response of the first and last pressure cycle of the loading regimen (Fig. 2). As before, the results are plotted for three specimens spanning the measured variation in the displacement response and were typical for all 10 specimens. The difference in the peak apex displacement between the first and last cycles was less than the mean error, Ei $=6.8 \pm 2.7 \mu \mathrm{m}$, of the DIC system. This result demonstrated that the deformation fully recovered between each pressure cycle and creep test, and the mechanical behavior of the tissue was not altered by the test conditions. 


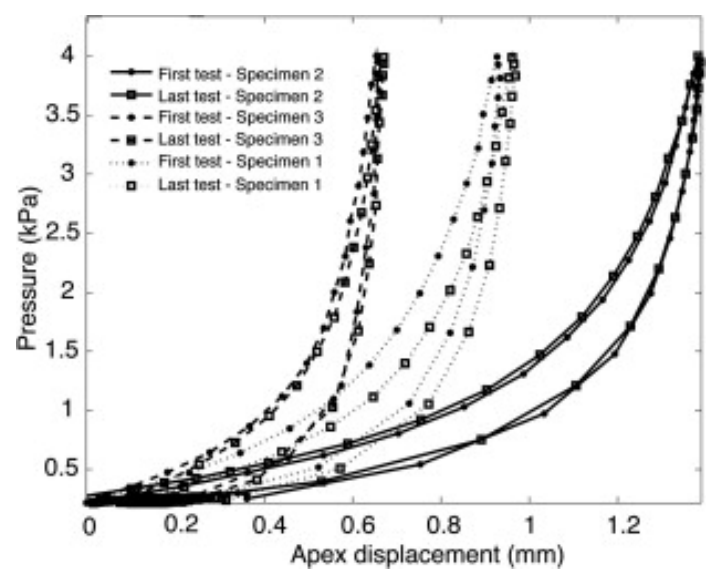

Fig. 7. Apex displacement response comparing the first and last pressure cycles of the loading regimen for three specimens. The tissue recovered completely between loadunload cycles and ramp-hold creep tests, indicating that the material properties of the tissue were not altered during testing.

\subsection{Rate-dependent displacement response}

Fig. 8 plots the specimen-averaged apex displacement response for the $0.13 \mathrm{kPa} \cdot \mathrm{s}-1$ pressure cycle to $4 \mathrm{kPa}$. Also plotted are the displacement responses for all 10 specimens to demonstrate the specimen variation. The variation between the samples was large and may have been caused by many factors, including differences in scleral dimensions, slaughter conditions and animal age. In general, the apex displacement response to pressure was nonlinear, with evidence of hysteresis during unloading to the baseline pressure. The average apex displacement at the peak pressure of $4 \mathrm{kPa}$ was $1.19 \mathrm{~mm}$.

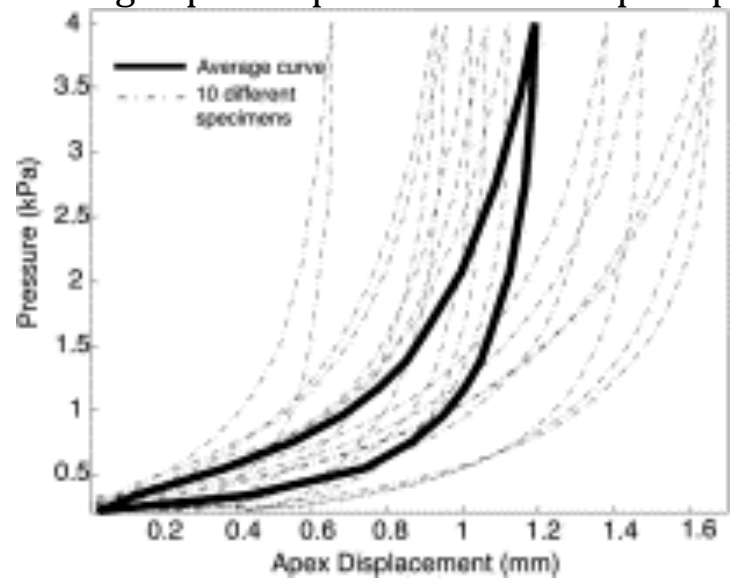

Fig. 8. The apex displacement response to the $0.13 \mathrm{kPa} \cdot \mathrm{s}^{-1}$ pressure cycle to $4.0 \mathrm{kPa}$ for 10 specimens (- ${ }^{-}$) and the specimen-averaged apex displacement response $(-)$.

The averaged apex displacement response for pressure cycles at three different rates is shown in Fig. 9. The tissue exhibited a rate-dependent behavior that is typical of viscoelastic materials. Both the apex displacement and the hysteresis decreased with increasing pressure rates for all the specimens tested. For each sample, we compared the displacement achieved at $4 \mathrm{kPa}$ after loading at the three different pressure rates. The differences in the response to the varying loading rates were compared to the standard 
deviation measured for the peak apex displacement responses for the three identical loadunload tests to ensure that the observed differences were not numerical artifacts. For each specimen tested, the difference in the displacement response between the different loading rates was found to be significantly larger than the difference in displacement response for the same loading rate. However, because of the large specimen-to-specimen variation, the differences in the averaged response with loading rate were not statistically significant.

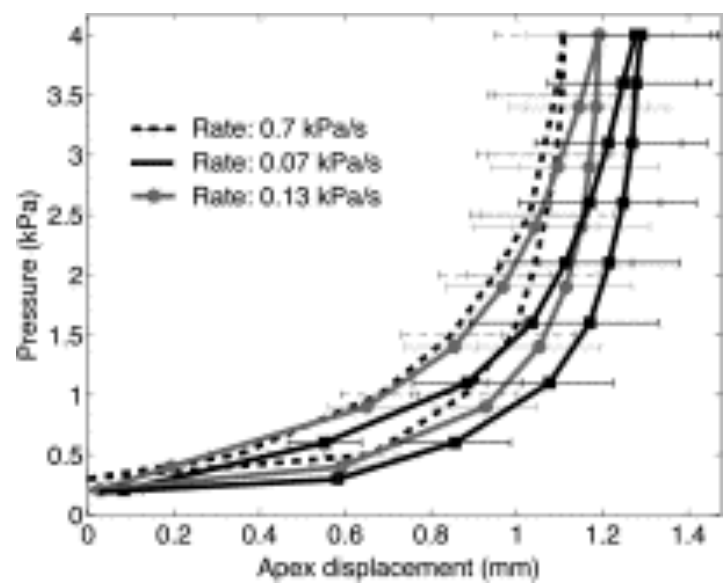

Fig. 9. The averaged apex displacement response to the load-unload tests at three different pressure loading rates. The sclera exhibited a rate-dependent displacement response.

\subsection{Creep}

Fig. 10 shows the averaged apex displacement response of the scleral specimens for the pressure-hold tests to 2, 4 and $6 \mathrm{kPa}$. In all the tests, the specimens displayed noticeable creep and the deformation recovered fully upon unloading. The recovery response did not depend on the applied pressure, and the three averaged recovery curves overlapped significantly. To examine the effect of the applied pressure on the creep rate, the averaged creep curves are plotted in Fig. 11. The creep rate of the three applied pressures showed slight differences, but these differences were not statistically significant (comparing the rates pair-wise yielded p-values $>0.05$ using a Student' $s$ t-test). This result suggests that the scleral tissue can be modeled as a quasilinear viscoelastic (QLV) material [27] and [28] in the physiological pressure range of 2-6 $\mathrm{kPa}(15-45 \mathrm{mmHg})$. The QLV model assumes a separable dependence of the stress response on strain and time, which allows the stress response to be calculated as the convolution of a time-dependent kernel and a nonlinear function of the strain. 


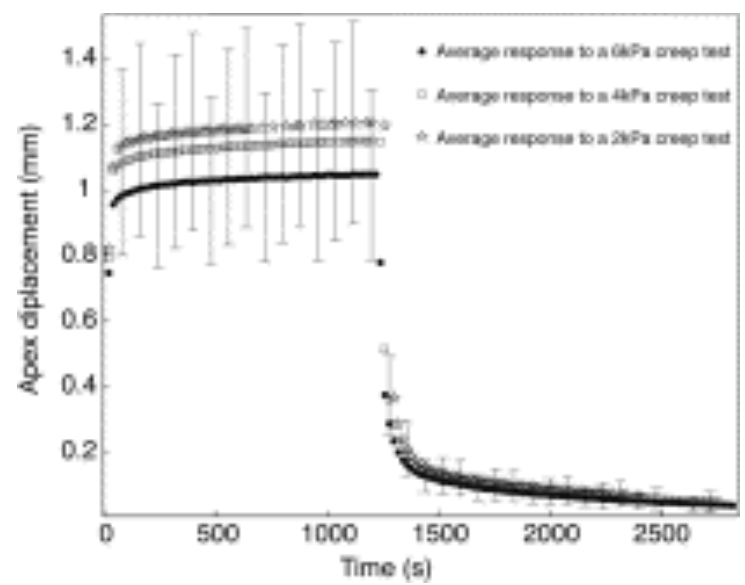

Fig. 10. The averaged creep response at three different applied pressures. The scleral specimens crept with time at all pressure levels during the 20 minute elevation hold, and the tissue specimens recovered completely within the 30 minutes of returning to the baseline pressure.

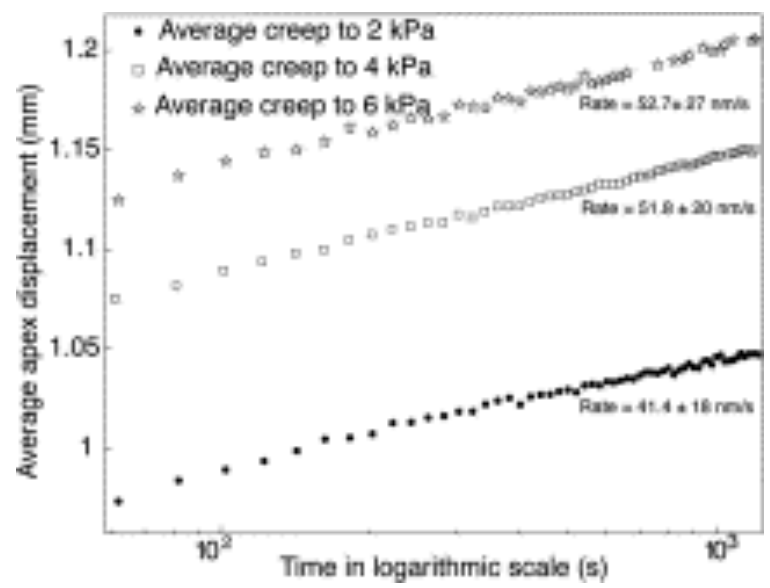

Fig. 11. The averaged creep rates at 15, 30 and $45 \mathrm{mmHg}$. The tolerances reported represent one standard deviation. There was no statistically significant difference between the measured creep rates (Student' s t-test, p > 0.05).

\section{Discussion}

A robust and repeatable in vitro inflation method was developed to measure the timedependent full-field deformation response of the sclera to inflation. In particular, the loading regimen was designed to study the effects of tissue preconditioning, ratedependency on the loading, pressure-dependency on the creep and tissue degradation during testing.

The method was demonstrated for bovine sclera. Results showed that the tissue exhibited a nonlinear and time-dependent inflation response to increases in pressure. The apex displacement response for the three initial repeated load-unload tests were nearly identical for each specimen, demonstrating that preconditioning of the intact tissue was unnecessary. Previous work showed that preconditioning was needed in uniaxial tests of the sclera [13], [14], [15] and [16] and the cornea [29] and [30] to obtain repeatable 
results. Further, our previous work [24] and that of Elsheikh and Anderson [30] on the cornea showed that the stress response measured by uniaxial strip tests was significantly stiffer than that measured by inflation tests. It can be hypothesized for the sclera and the cornea that preconditioning causes the lamellae to reorient towards the loading axis, resulting in large permanent deformation and dramatic stiffening of the stress response of the tissue. Consequently, the mechanical behavior reported for uniaxial strip tests is less representative of the behavior in vivo than that reported by inflation tests, which preserve the structure of the tissue and do not require preconditioning. Results in this study also showed that the inflation response of the tissue did not degrade during the hours of testing. The difference in the apex displacement response for the first and last load-unload tests was within the error of the test, confirming that the mechanical testing regimen did not alter the material properties of the tissue during the testing.

The inflation response of the bovine sclera to elevations in pressure was viscoelastic, showing rate-dependence, hysteresis and creep. The time-dependent behavior observed in the results suggests that the deformation and stress state of the sclera and the attached $\mathrm{ONH}$ may be influenced by dynamic fluctuations in IOP along with the baseline IOP level [31]. In addition, recent in vivo IOP monitoring of monkeys revealed that these dynamic IOP fluctuations might fall within a time scale of when the material response of the sclera tissue is time-dependent [32]. Therefore, to more accurately assess the stress environment of the $\mathrm{ONH}$ and the cumulative damage it may cause to the RGCs over time, viscoelastic scleral properties must be taken into account.

Along with the tissue being viscoelastic, the data presented here suggest that the bovine scleral material properties vary regionally. The 3-D displacement field of the inflating scleral surface revealed that the location of the maximum displacement was off-centered. The largest displacements were measured around the $\mathrm{ONH}$ in the peripapillary sclera. This scleral displacement pattern may correspond to a collagen/elastin network preferentially aligned in the circumferential direction around the $\mathrm{ONH}$. The circumferential reinforcement produces a stiff circumferential orientation, which reduces the hoop expansion of the adjacent $\mathrm{ONH}$ and results in a more compliant vertical orientation, as observed in the inflation experiments. More evidence is needed to confirm that the dominant region of scleral deformation extends around the $\mathrm{ONH}$ for the bovine sclera. For example, an additional stereoscopic DIC set-up can be employed to capture inflation below the scleral equator. Further, histological and finite element modeling studies will be needed to determine the regional material properties and the preferential collagen/elastin network alignment of the bovine sclera. We are currently developing a constitutive model that includes the anisotropic fiber structure, and we plan to fit this model to the 3-D DIC measured displacement field to obtain the viscoelastic properties of the tissue. This modeling effort will be presented in a future study. In addition, we are applying the in vitro inflation test to measure and compare the mechanical properties of human sclera of normal and glaucomal eyes to study the influences of scleral biomechanics in the development of glaucoma. 
There are limitations to our study and we are working to improve the methodology. The thickness of the bovine sclera was measured only at the apex and a point at the equator using digital calipers at the end of the test. We are developing methods to measure the thickness more accurately and at more points on the specimen using pachymetry and microscopy. For human scleral specimens, pachymetry works well because the thickness for the human specimens is within the range of the ultrasound probe. However, the bovine sclera was too thick to use pachymetry. We validated our caliper measurements by conducting a few measurements using a dissecting microscope. The differences between the thickness measurements using calipers and a dissecting microscope were within the standard deviation reported in Table 1. The specimen-to-specimen variation in the displacement measurements was large, such that we were unable to measure a statistically significant variation with loading rate among the 10 specimens. Variations in the specimen geometry (e.g. thickness and axial length) were likely significant contributors to the experimental scatter. We are currently developing specimen-specific finite element models of the posterior sclera to analyze the inflation data and account for the effects of geometric variations. The experiments were not conducted using in vivo temperature conditions and boundary conditions. Testing at an elevated temperature, without in vivo mechanisms to maintain collagen integrity, would have accelerated tissue degradation. Testing at room temperature provides for more robust viscoelastic measurements and suffices for the purpose of comparing the differences in the viscoelastic behavior of normal and diseased tissue. The in vitro creep rates reported in this study overestimated the creep rates experienced in vivo. Nonetheless, in vitro creep rates can be productively compared between normal and diseased tissue to gain an understanding of the baseline differences in viscoelastic properties. Lastly, we chose to present the scleral displacement profiles because of the difficulties of calculating strains analytically, posed by the presence of the $\mathrm{ONH}$ and large thickness variations, and using the DIC algorithm caused by irregularities of the scleral surface.

\section{Conclusions}

Inflation tests were performed on bovine sclera to characterize the viscoelastic response of the tissue to controlled pressurization. The inflation test used stereoscopic digital image correlation to obtain a 3-D displacement field of the deforming scleral surface, which is important to measure the spatially varying material properties of the tissue. The results showed that the maximum magnitude of the displacement was localized in the region surrounding the $\mathrm{ONH}$. This agrees with histological studies showing a strong circumferential collagen/elastin arrangement in the peripapillary region, which leads to a stiff circumferential orientation but compliant radial orientation. The creep rates observed during the constant pressure tests were not statistically affected by the pressure level, which suggests that the viscoelastic behavior of the tissue may be adequately described by quasilinear viscoelastic models. Bovine sclera was used for the protocol development because of the availability and consistency of the tissue. The ultimate goal of this research is to investigate the mechanical behavior of the human sclera and the role of scleral mechanics in the development of diseases and conditions such as glaucoma and myopia. Consequently, our current work extends these testing methods to human sclera. Human eye bank tissue specimens are prepared and inflated in a similar manner to the bovine specimens to compare the viscoelastic properties of the sclera of normal and glaucomal 
eyes. The results will be used to develop a viscoelastic constitutive model that incorporates the effects of the anisotropic collagen/elastin structure and finite element models of the posterior sclera. The models will be applied to study how the variations in the tissue structure and mechanical properties influence the development of glaucoma and to predict the outcomes of therapeutic intervention.

Acknowledgements

The authors thank Harry Quigley and Russ McCally of the Wilmer Eye Institute at Johns Hopkins Medical Institute for helpful discussions on the biomechanics of the sclera and glaucoma.

References

[1] M. Hogan, J.A. Alvarado, J. Weddell. Histology of the human eye. W.B. Saunders Company, Philadelphia, PA (1971) p. 193-9.

[2] J. Rada, S. Shelton, T. Norton. The sclera and myopia. Exp Eye Res, 82 (2) (2006), pp. 185-200.

[3] S. Gelman, F.E. Cone, M.E. Pease, T.D. Nguyen, K. Myers, H.A. Quigley. The presence and distribution of elastin in the posterior and retrobulbar regions of the mouse eye. Exp Eye Res, 90 (2) (2010), pp. 210-215.

[4] H.A. Quigley, M.E. Dorman-Pease, A. Brown. Quantitative study of collagen and elastin of the optic nerve head and sclera in human and experimental monkey glaucoma. Curr Eye Res, 10 (9) (1991), pp. 877-888.

[5] P.G. Watson, R.D. Young. Scleral structure, organization and disease. A review. Exp Eye Res, 78 (3) (2004), pp. 609-623.

[6] A. Whitmore, R. Libby, S. John. Glaucoma: thinking in new ways - a role for autonomous axonal self-destruction and other compartmentalized processes. Prog Retin Eye Res, 24 (6) (2005), pp. 639-662.

[7] C.F. Burgoyne, J.C. Downs, A. Bellezza, J.K.F. Suh, R.T. Har. The optic nerve head as a biomechanical structure: a new paradigm for understanding the role of IOP-related stress and strain in the pathophysiology of glaucomatous optic nerve head damage. Prog Retin Eye Res, 24 (1) (2005), pp. 39-73.

[8] R. Nickells. From ocular hypertension to ganglion cell death: a theoretical sequence of events leading to glaucoma. Can J Ophthalmol, 42 (2) (2007), pp. 278-287.

[9] C.R. Ethier. Scleral biomechanics and glaucoma - a connection?. Can J Ophthalmol, 41 (1) (2006), p. 9. 
[10] J. Phillips, M. Khalaj, N. McBrien. Induced myopia associated with increased scleral creep in chick and tree shrew eyes. Invest Ophthalmol Vis Sci, 41 (8) (2000), pp. 20282034.

[11] J. Phillips, N. McBrien. Form deprivation myopia: elastic properties of sclera. Ophthalmic Physiol Opt, 15 (5) (1995), pp. 357-362.

[12] P. Greene, T. McMahon. Scleral creep vs. temperature and pressure in vitro. Exp Eye Res, 29 (5) (1979), pp. 527-537.

[13] D.S. Schultz, J.C. Lotz, S.M. Lee, M.L. Trinidad, J.M. Stewart. Structural factors that mediate scleral stiffness. Invest Ophthalmol Vis Sci, 49 (10) (2008), pp. 4232-4236.

[14] M.J.A. Girard. Effects of storage time on the mechanical properties of rabbit peripapillary sclera after enucleation. Curr Eye Res, 32 (5) (2007), pp. 465-470.

[15] J.C. Downs, J.K.F. Suh, K. Thomas, A. Bellezza, R.T. Hart, C.F. Burgoyne. Viscoelastic material properties of the peripapillary sclera in normal and early-glaucoma monkey eyes. Invest Ophthalmol Sci, 46 (2) (2005), pp. 540-546.

[16] J.C. Downs, J.K.F. Suh, K. Thomas, A. Bellezza, C.F. Burgoyne, R.T. Hart. Viscoelastic characterization of peripapillary sclera: material properties by quadrant in rabbit and monkey eyes. J Biomech Eng, 125 (1) (2003), pp. 124-132.

[17] T. Friberg, J. Lace. A comparison of the elastic properties of human choroid and sclera. Exp Eye Res, 47 (3) (1988), pp. 429-436.

[18] B.J. Curtin. Physiopathologic aspects of scleral stress-strain. Trans Am Ophthalmol Soc, 67 (1969), pp. 417-461.

[19] M.J.A. Girard, J.K.F. Suh, M. Bottlang, C.F. Burgoyne, J.C. Downs. Scleral biomechanics in the aging monkey eye. Invest Ophthalmol Vis Sci, 50 (11) (2009), pp. 5226-5237.

[20] M.J.A. Girard, J.C. Downs, C.F. Burgoyne, J.K.F. Suh. Experimental surface strain mapping of porcine peripapillary sclera due to elevations of intraocular pressure. J Biomech Eng, 130 (2008), p. 041017.

[21] S. Woo, A. Kobayashi, W. Schlegel, C. Lawrence. Nonlinear material properties of intact cornea and sclera. Exp Eye Res, 14 (1) (1972), pp. 29-39.

[22] A. Hommer, G. Fuchsjager-Mayrl, H. Resch, C. Vass, G. Garhofer, L. Schmetterer. Estimation of ocular rigidity based on measurements of pulse amplitude using pneumotonometry and fundus pulse using laser interferometry in glaucoma. Invest Ophthalmol Vis Sci, 49 (2008), pp. 4046-4050. 
[23] J.R. Phillips, N.A. McBrien. Pressure-induced changes in axial eye length of chick, tree shrew: significance of myofibroblasts in the sclera. Invest Ophthalmol Vis Sci, 45 (3) (2004), pp. 758-763.

[24] B. Boyce, J. Grazier, R. Jones, T.D. Nguyen. Full-field deformation of bovine cornea under constrained inflation conditions. Biomaterials, 29 (28) (2008), pp. 3896-3904.

[25] G.G. Gum, K.N. Gelatt, D.N. Miller, E.O. Mackay. Intraocular pressure in normal dairy cattle. Vet Ophthalmol, 1 (1998), pp. 159-161.

[26] H.W. Schreier. Advances in light microscope stereo vision; experimental mechanics. Exp Mech, 44 (3) (2004), pp. 278-288.

[27] Y.C. Fung. Biomechanics: mechanical properties of living tissues. Springer-Verlag, New York (1993) p. 277.

[28] P. Provenzano, R. Lakes, T. Keenan, R. Vanderby. Nonlinear ligament viscoelasticity. Ann Biomed Eng, 29 (10) (2001), pp. 908-914.

[29] B. Boyce, R. Jones, T.D. Nguyen, J. Grazier. Stress-controlled viscoelastic tensile response of bovine cornea. J Biomech, 40 (11) (2007), pp. 2367-2376.

[30] A. Elsheikh, K. Anderson. Comparative study of corneal strip extensometry and inflation tests. J R Soc Interface, 2 (3) (2005), p. 177.

[31] C.F. Burgoyne, H. Yang, I. Sigal, M. Roberts, H. Thompson, J.C. Downs. Deformation of the normal monkey optic nerve head $(\mathrm{ONH})$ connective tissues following acute IOP elevation within 3-D histomorphometric reconstructions. Invest Ophthalmol Vis Sci, 50 (2009) E-Abstract 4897.

[32] J.C. Downs, C.F. Burgoyne, Y. Liang, V.L. Sallee. Telemetric IOP monitoring in the adult monkey shows ocular pulse varies with IOP. Invest Ophthalmol Vis Sci, 50 (2009) EAbstract 2842. 